\title{
Erythrocyte Efferocytosis by the Arterial Wall Promotes Oxidation in Early-Stage Atheroma in Humans
}

\author{
Sandrine Delbosc ${ }^{1,2 t}$, Richard Graham Bayles ${ }^{1,2 t}$, Jamila Laschet ${ }^{1,2}$, Veronique Ollivier ${ }^{1,2}$, \\ Benoit Ho-Tin-Noé1,2, Ziad Touat ${ }^{1,2}$, Catherine Deschildre ${ }^{1,2}$, Marion Morvan ${ }^{1,2}$, \\ Liliane Louedec 1,2, Laurent Gouya ${ }^{2,3}$, Kevin Guedj ${ }^{1,2}$, Antonino Nicoletti',2 \\ and Jean-Baptiste Michel, ${ }^{1,2 *}$
}

\begin{abstract}
${ }^{1}$ UMRS 1148, INSERM, Paris 7-Denis Diderot University, Hôpital Xavier Bichat, Paris, France, ${ }^{2}$ Département HospitaloUniversitaire DHU "FIRE", Paris, France, ${ }^{3}$ UMRS 1149, INSERM, Paris 7-Denis Diderot University, Hôpital Xavier Bichat, Paris, France
\end{abstract}

Background: Since red blood cells (RBCs) are the predominant cellular blood component interacting with the arterial wall, we explored the role of RBCs efferocytosis by vascular smooth muscle cells (vSMCs) in the initiation of human atheroma.

OPEN ACCESS

Edited by:

Masataka Sata,

Tokushima University, Japan

Reviewed by:

Jozsef Balla,

University of Debrecen, Hungary

Adriana Georgescu,

Institute of Cellular Biology and

Pathology (ICBP), Romania

*Correspondence:

Jean-Baptiste Michel jean-baptiste.michel@inserm.fr

these authors have contributed equally to this work.

Specialty section:

This article was submitted

to Atherosclerosis and

Vascular Medicine,

a section of the journal

Frontiers in Cardiovascular Medicine

Received: 13 April 2017

Accepted: 26 June 2017

Published: 02 August 2017

Citation:

Delbosc S, Bayles RG, Laschet J, Ollivier V, Ho-Tin-Noé B, Touat Z, Deschildre C, Morvan M, Louedec L,

Gouya L, Guedj K, Nicoletti A and Michel J-B (2017) Erythrocyte

Efferocytosis by the Arterial Wall

Promotes Oxidation

in Early-Stage Atheroma in Humans.

Front. Cardiovasc. Med. 4:43.

doi: 10.3389/fcvm.2017.00043
Methods and results: The comparison of human healthy aortas with aortic fatty streaks or fibroatheromas revealed that RBC angiophagy is implicated from the earliest stages of atherogenesis, as documented by the concomitant detection of redox-active iron, hemoglobin, glycophorin A, and ceroids. RBCs infiltration in the arterial wall was associated with local lipid and protein oxidation, as well as vascular response (expression of heme oxygenase- 1 and of genes related to iron metabolism as well as those encoding for phagocytosis). These effects were recapitulated in vitro when vSMCs were co-cultured with phosphatidyl-exposing senescent (s) RBCs but not with fresh RBCs. VSMCs engulfing SRBC increased their intracellular iron content, accumulated hemoglobin, lipids, and activated their phagolysosomes. Strikingly, injections of sRBCs into rats promoted iron accumulation in the aortic wall. In rabbits, hypercholesterolemia increased circulating senescent RBCs and induced the subendothelial accumulation of iron-rich phagocytic foam cells. RBCs bring cholesterol and iron/heme into the vascular wall and interact with vSMCs that phagocytize them.

Conclusion: This study presents a previously unforeseen mechanism of plaque formation that implicates intimal RBC infiltration as one of the initial triggers for foam cell formation and intimal oxidation. Pathogenic effects exerted by several metabolic and hemodynamic factors may rely on their effect on RBC biology, thereby impacting how RBCs interact with the vascular wall.

Keywords: smooth muscle cells, oxidative stress, iron, fatty streaks, hemoglobin, translational studies, vascular biology, atherosclerosis

\section{INTRODUCTION}

Atherothrombosis is a consequence of multiple interactions between blood circulating components and the arterial wall. These initial interactions involve both radial convection and intimal retention of low-density lipoprotein (LDL) (1), and collision of blood cells [platelet, red blood cell (RBC), and leukocytes] with the wall (2). Such interactions are greatly potentiated by local hemorheology 
modified by non-uniform geometries of the endoluminal wall surface (3-5), which could explain the focal nature of the disease. Intimal oxidative processes characterize early human and rabbit atheroma (6), but the triggers of these remain uncertain and can result in site-specific injuries of the intima caused by circulating blood cells.

In previous and recent human (7-9) and murine (10-12) studies, intimal vascular smooth muscle cells (vSMCs) were identified as important contributors to the foam cells and phagocyte contingents to the atherosclerotic wall. vSMCs from the tunica media migrate into the intima and respond to initial lipid and cellular injuries by engaging their clonality (12) and phenotypic plasticity (13). The roles of leukocytes (14) and platelets (15) in the initiation of atherothrombosis have been extensively investigated. Despite their high predominance as circulating cells, the roles of RBCs and of their hemoglobin cargo have been less explored (16). Nevertheless, it has been demonstrated that free heme by increasing granulocyte ROS production (17) or LDL oxidation (18) participated to the endothelial injury. This point has also been confirmed with free hemoglobin cytotoxicity for the arterial wall (19). However, the direct role of RBC collision and penetration within the arterial wall and its impact on resident vSMCs behind the endothelium have not yet described in human atherosclerotic tissue.

Key studies reported by the groups of Arbustini et al. (20) and Kolodgie (21) have demonstrated that intraparietal RBCs, through intraplaque hemorrhages and hematoma, can also critically contribute to lipid core expansion in relation to their membrane cholesterol content. These observations were then expanded to oxidation (22). Due to their phenotypic plasticity, human vSMCs possess both endocytic (8) and efferocytic (23) capacities, which are associated with atheroma development. vSMCs have been reported to transform into foam cells when they internalize macromolecules, such as aggregated or oxidized LDL (24), or microparticles (25). Importantly, we have reported that this is also the case following the engulfment and intracellular metabolism of senescent RBCs (sRBCs) (23).

In the present study, we studied how the interactions between RBCs, and the wall intima could impact the early stages of human atheroma. To accomplish this, we evaluated intraparietal iron and hemoglobin content and tissue-associated oxidative stress in human fatty streaks (FSs) and fibro-atheroma. Iron retention was found to be linked to the metabolism of RBC components predominantly achieved by vSMCs. We tested the ability of cultured human vSMCs to perform erythrophagy in vitro, and analyzed its consequences on lipophagy, iron retention, and oxidation. We further confirmed this relationship in vivo using the foam cell-rich hypercholesterolemic rabbit model, and a rat model created by subjecting animals to repeated intravenous injections of sRBCs.

\section{MATERIALS AND METHODS}

\section{Tissue Sampling}

Human aortas were consecutively collected from deceased organ donors from 2010 to 2013 under the authorization of the
French Biomedicine Agency (PFS 09-007). After macroscopic examination, the aortas were classified according to Stary classification (26) and the Virmani word list (27) into three groups: healthy aortas (HAs, $n=34)$, aortas with FS $(n=67)$, and aortas with fibrolipidic initial plaques [fibroatheromas (FAs), $n=56$ ]. A small portion of tissue from each sample was fixed in $3.7 \%$ paraformaldehyde for classical histology and immunochemistry assessments. For the HA samples, it was practically and virtually impossible to separate and independently process the tunica intima. For these samples, the adventitia was carefully removed, and only the results obtained from the tunica media are presented. For the FS and FA samples, the adventitia was also carefully removed, and the dissected intimal lesions and media layers were processed separately. Tunica medias from HA, FS, and FA, as well as intimal lesions from FS and FA were either incubated in RPMI-1640 culture medium $\left(24 \mathrm{~h}, 37^{\circ} \mathrm{C}\right)$ to obtain tissue-conditioned media or placed in RNALater $\left(24 \mathrm{~h}, 4^{\circ} \mathrm{C}\right)$ for RNA extraction. The isolated tunica intimas (FS $n=8$, FA $n=8$ ) were directly fixed in paraformaldehyde for subsequent "whole mount" immunohistochemistry.

\section{Human Vascular SMC Culture}

Vascular smooth muscle cells were isolated from human HAs. As previously described (28), the tunica media was separated from the tunica adventitia before being cut into small pieces and digested in collagenase/elastase. Cells were subcultured to the sixth passage prior to experimentation. Purity of the culture was assessed by immunostaining of SMC $\alpha$-actin and transgelin (SM22- $\alpha$ ) (data not shown).

\section{Experimental Models for RBC-Triggered Arterial Wall Injuries}

\section{Rabbit Model}

Five New Zealand white rabbits were fed a high-fat diet $(4.7 \%$ coconut oil and $0.3 \%$ cholesterol-enriched diet, Research Diet) for 6 weeks as previously described $(29,30)$. The rabbits were sacrificed under deep anesthesia $(50 \mathrm{mg} / \mathrm{kg}$, IP), and their aortas were removed and fixed in $3.7 \%$ paraformaldehyde.

\section{Rat Model}

Thirty-eight inbred male Lewis rats were treated with two intravenous injections $\left(2 \times 10^{8}\right.$ cells in $500 \mu \mathrm{l}, 2$ and 4 days after surgery) of sRBCs (see below) isolated from syngeneic Lewis rats. Controls $(n=38)$ were injected with the same volume of saline. Prior to RBC administration, 20 rats in each of these groups underwent a balloon injury of the infrarenal abdominal aorta or a sham operation $(n=18)$. In each of these subsets, the rats either underwent splenectomy $(n=10)$ or did not $(n=8)$. The surgical procedure was as follows. After intraperitoneal administration of pentobarbital anesthesia, a median laparotomy was performed, and the left iliac artery was isolated and ligated. The aorta was then isolated and clamped below the renal arteries. A 3F Fogarty balloon catheter was inserted by the right iliac artery, pushed upwards into the aorta, and inflated. The inflated balloon was gently removed three times, causing injury to the abdominal aorta, as previously described (31). Splenectomy was 
performed by ligature of the spleen vascular pedicles. 10 days after surgery, the rats were anesthetized with sodium pentobarbital (50 mg/kg, IP) and sacrificed. Their aortas were removed and either fixed in $3.7 \%$ paraformaldehyde or snap-frozen for iron content determination.

All experimental and animal care procedures complied with the principles formulated by the National Society for Medical Research (animal facility agreement: No. B75-18-03). This study was performed under the advice of our institution's animal ethical committee (authorization no. 75-101) and complied with all guidelines for animal experimentation set forth by the French Ministry of Agriculture.

\section{Senescent RBCs}

Peripheral blood was collected from healthy volunteers and Lewis rats in heparinized tubes. Following this, the blood was centrifuged at $120 \mathrm{~g}$ for $15 \mathrm{~min}$, and the plasma and the buffy coat were removed. The pellet containing RBCs was then mixed (1:1) with a dextran solution (2\% in HBSS) for $15 \mathrm{~min}$, centrifuged (1,500 $\mathrm{g}$ for $5 \mathrm{~min}$ ), and washed four times with HBSS (with $\mathrm{Ca}^{2+}$ and $\mathrm{Mg}^{2+}$ ) supplemented with $1 \%$ antibiotic. Finally, to obtain sRBCs, the pellet was resuspended in HBSS supplemented with $0.1 \%$ glucose and incubated at $37^{\circ} \mathrm{C}$ for 5 days. RBCs incubated in HBSS without glucose (fRBCs) were used as controls. RBC senescence was validated by evaluating phosphatidylserine (PS; annexin V-FITC binding) exposure on cell membranes by flow cytometry. RBCs were washed with PBS before in vivo (rats) or in vitro (cell biology) use in order to limit the direct effect of free hemoglobin.

\section{Tissue Hemoglobin Content Oxidative Stress Markers, Glycophorin A, and Iron Content}

Hemoglobin content was estimated by the formic acid method. The acid induced $\mathrm{CO}$ (carbon monoxide) formation which reacted with hemoglobin to create a reversible carboxy-hemoglobin. $30 \mu \mathrm{L}$ of the samples or the standards and $70 \mu \mathrm{L}$ of formic acid were added to 96-well plates. Absorbance was read immediately at $420 \mathrm{~nm}$. Hemoglobin values were calculated based on the standard curve (free hemoglobin).

Thiobarbituric acid reactive substance (TBARS), AOPPs (markers of oxidized lipids and proteins, respectively) (28), and Gly-A concentrations (ELISA kit from USCNK) were determined in the conditioned medium of human atherosclerotic lesions. To determine iron content, tissue samples were incubated for $48 \mathrm{~h}$ in an extraction solution containing $6.25 \mathrm{~mL} \mathrm{H}_{2} \mathrm{O}, 1.25 \mathrm{~g}$ trichloroacetic acid, $3.225 \mathrm{~mL} \mathrm{36 \%} \mathrm{HCl}$, and $0.375 \mathrm{~mL} 98 \%$ thioglycolic acid. The samples were then diluted 1:10 in $1.5 \mathrm{M}$ sodium acetate. Iron concentrations were determined based on spectrophotometric readings taken at $535 \mathrm{~nm}$ and expressed as $\mathrm{mM} / \mathrm{mg}$ of tissue.

\section{Incubation of RBCs and $\mathrm{Hb}$ with vSMCs and ROS Production}

Intracellular ROS production by vSMCs was determined using 5and 6-chloromethyl-2,7'-dichlorodihydrofluorescein diacetate, acetyl ester (CM-H2DCFDA, Life Technologies) (28). vSMCs were seeded in a 96-well plate and starved overnight after reaching $80 \%$ confluence. Senescent or fresh RBCs $\left(1 \times 10^{6}\right.$ cells $)$ were added to the vSMCs and incubated for 3 days. In separate experiments, vSMCs were incubated with increasing concentrations of $\mathrm{Hb}(0-100 \mu \mathrm{M})$, in the presence or the absence of desferoxiamine $(20 \mu \mathrm{M})$. After washing the vSMCs, $5 \mu \mathrm{M}$ CM-H2DCFDA was added to each well, and fluorescence intensity was monitored every $2 \mathrm{~min}$ for $2 \mathrm{~h}$. The results are expressed as oxidation rates (fluorescence intensity/min; mean \pm SEM).

\section{Phagocytosis Assay}

Vascular smooth muscle cells were seeded and grown in a 6-well plate until $80 \%$ confluent. Fresh or senescent RBCs $\left(1 \times 10^{6}\right.$ cells $)$ were labeled with CFSE according to the manufacturer's instructions and incubated with the vSMCs for 3 days. At the end of the experiment, the supernatant was removed, and the cells were washed three times in PBS and dissociated using trypsin. After being centrifuged $(600 \times g, 5 \mathrm{~min})$, the cells were resuspended in $4 \%$ PFA and analyzed at $40 \times$ magnification on an ImageStream flow cytometer. In brief, images of vSMCs that had been cultured in the presence of fresh or senescent RBCs were used for measuring the internalization coefficient (internalization function of the IDEAS $^{\circledR}$ analysis software from Amnis). The software calculates a ratio based on the fluorescence intensity inside the cell compared with the fluorescence intensity of the entire cell. To discriminate both signals, we defined a mask smaller than the cell in which internalization was measured. An elevated ratio indicates a high internalization coefficient.

\section{Tissue and Cell RT-PCR}

Aortic tunica intima and media tunicas were independently cryopulverized in liquid nitrogen using a freezer mill (model 6750 SPEX SamplePrep). Total RNA was extracted from the pulverized tissues and vSMCs using TRIzol reagent (Life Technologies) according to the manufacturer's protocol. Reverse transcription was performed using the MMLV enzyme (Life Technologies), and real-time PCR was conducted in a LightCycler system using a SYBR green detection kit (Roche Applied Science) with specific primers (Table 1).

\section{Histology and Immunohistochemistry}

Aortic tissues were embedded in paraffin and cut into $5-\mu \mathrm{m}$ thick sections. For their morphological characterization, we used hematoxylin and eosin and Masson's trichrome staining. DiAminoBenzidin (DAB) staining was used to determine the presence of peroxidase activity (32). Ferric iron (FeIII) storage (present mostly in association with ferritin) was visualized by the ferrocyanide precipitation (Perl's Prussian blue staining). Nevertheless, Prussian blue precipitation is highly specific for FeIII storage, but poorly sensitive to detect other forms of redoxactive iron. Therefore, the presence of the low-molecular-weight iron pool (33) and of highly redox-active Fe (34) was assessed by the combination of Perls and DAB staining, successively associating two redox pairs, $\mathrm{Fe}^{+++} / \mathrm{Fe}^{++}$(Perls) and $\mathrm{H}_{2} \mathrm{O}_{2} / \mathrm{H}_{2} \mathrm{O}$ leading to $\mathrm{DAB}$ polymerization by oxidation. In these reactions, endogenous oxidases were not primarily blocked, since redox-active iron pair catalyze endogenous oxidase reactions (Haber-Weiss and Fenton 
TABLE 1 | Primers used for real-time PCR.

\begin{tabular}{|c|c|c|}
\hline & Forward & Reverse \\
\hline $\mathrm{HO}-1$ & 5’ AACTाTCAGAAGGGCCAGGT 3' & 5' TGTTGCGCTCAATCTCCTC 3' \\
\hline NRAMP-1 & 5’ ATGACAGGTGACAAGGGTCC3' & 5' GGGCTGGAGATGGAACCATAG 3' \\
\hline Ferritin & 5' CTGGAGCTCTACGCCTCCTA 3' & 5' CCACATCATCGCGGTCAAAG 3' \\
\hline TIM-3 & 5' TCCAAGGATGCTTACCACCAG 3' & 5' GCCAATGTGGATATTGTGTTAGATT 3' \\
\hline STAB1 & 5' TCAAGTCGCTGCCTGCATAG 3' & 5' CAGCGTGCCAAAGAAACCAG 3' \\
\hline MR & 5' GACCAAAAGGCCAGAAAGGG 3' & 5' CAAGCTCCTACAGACGACCT 3' \\
\hline HPRT & 5' TGAGGATITGGAAAGGGTGT 3' & 5' GAGCACACAGAGGGCTACAA 3' \\
\hline
\end{tabular}

reactions) detected by DAB polymerization (32). Negative controls are $\mathrm{DAB}$ alone and Perls alone.

To detect the presence of fluorescent ceroids ( $\lambda$ excitation 510-560 nm, $\lambda$ emission $590 \mathrm{~nm}$ ), fixed sections were observed using a Zeiss Axiovert $200 \mathrm{M}$ inverted microscope equipped with an AxioCam MRm version 3 camera, an ApoTome ${ }^{\circledR}$ system and AxioVision ${ }^{\circledR}$ image capture software. In addition, positive Oil Red O (ORO) staining indicated ceroid ability to retain lipids [persistent staining despite delipidation during paraffin removal by detergents (35)]. Fluorescence measurements taken at 330-380 nm (U.V.) were used to compare sections with or without ORO staining of ceroids. Gly-A (Dako) immunostaining was used to visualize RBC membranes on paraffin sections of early atheroma lesions. Immunostaining of smooth muscle cell-specific myosin (MYH-11) was performed using the monoclonal antibody, ID8 (EMD, Millipore). The immunodetection of hemoglobin was performed using a rabbit polyclonal antibody (H4890, Sigma).

Because no antibody that recognizes rabbit Gly-A is currently available, we used Bandeiraea simplicifolia isolectin B4 (GSLB4, L2140, $10 \mu \mathrm{g} / \mathrm{ml}$ Sigma Chemical), a previously described ligand of rabbit RBC membrane glycoconjugates bearing $\alpha$-Dgalactose (30). Rabbit phagocytes were detected using a mouse monoclonal anti-rabbit RAM11 antibody [M063, dilution 1:500, Dako North America Inc. (29)]. These immunostaining experiments were performed on paraformaldehyde-fixed rabbit aorta cross-sections. A horseradish peroxidase-conjugated secondary antibody and DAB were used to reveal the primary antibody.

\section{Statistics}

All the results are expressed as the mean \pm SEM of multiple experiments. Statistical analyses were performed using Prism 5 (GraphPad software). Differences in event frequencies were assessed using chi-squared tests. Differences between two groups were assessed with Mann-Whitney tests and were considered significant at $p<0.05$.

\section{RESULTS}

\section{Iron and Hemoglobin Deposits Are Present in Early Atherosclerotic Human Aortas}

We first evaluated the presence of iron deposits in consecutively collected human FSs and FAs or in human aortas without atherosclerotic lesions (HAs). The average age of the patients with FAs
TABLE 2 | Stages of early lesions in the thoracic and abdominal aortas according to subject age and gender.

\begin{tabular}{lccc}
\hline & HA ( $\boldsymbol{n}=\mathbf{3 4})$ & FS $(\boldsymbol{n}=\mathbf{6 7})$ & FA ( $\boldsymbol{n}=\mathbf{5 6})$ \\
\hline Age (years) & $48 \pm 17$ & $53 \pm 17$ & $63 \pm 12^{\star \star}$ \\
Gender (\% male) & $69 \%$ & $59 \%$ & $68 \%$ \\
Thoracic aorta & $62 \%^{\star \star \star}$ & $14 \%$ & $24 \%$ \\
Abdominal aorta & $32 \%$ & $25 \%$ & $43 \%$ \\
Perls' + DiAminoBenzidin (DAB) & $22 \%^{\star \star \star}$ & $84 \%$ & $90 \%$ \\
DAB (alone) & $0 \%$ & $16 \%$ & $26 \%$ \\
Perls' (alone) & $0 \%$ & $9 \%$ & $39 \%$ \\
Ceroid detection & $23 \%^{\star \star \star}$ & $71 \%$ & $94 \%$
\end{tabular}

The frequencies with which iron and ceroids were detected are listed at the bottom of the table.

${ }^{* *} p<0.01,{ }^{* * *} p<0.0001$, chi-squared test.

was significantly higher $(p<0.001)$ than that with HAs. The male gender was predominant regardless of aorta status $(p<0.001)$. HAs were more frequent in the thoracic tract than in the abdominal tract $(p<0.001$, Table 2).

As depicted in Figure 1, HAs were macroscopically characterized by the presence of a smooth, regular endovascular surface, whereas diseased aortas displayed FSs that resembled thin yellow deposits that were always localized on the dorsal portion of the aorta, facing the intercostal/lumbar artery ostia. In the assessed FA lesions, white capsules were found surrounding the yellow deposits (Figure 1, bottom row). Histologically, the FSs were composed of a large number of cells, whereas the lipid centers of the FAs were acellular and devoid of nuclei (Figure 2A).

Masson's trichrome staining (Figure 1) showed that the FS and FA samples were characterized by the presence of foam cells, resulting in a translucent or granular cytosolic appearance. Co-staining with Perls + DAB revealed that granular foam cells in the FS and FA samples accumulated redox active iron. Perls alone and $\mathrm{DAB}$ alone are strictly negative. In the HA samples, only a few focal iron deposits were observed (Figure 1). $147 \mathrm{sec}$ tions (each from a different donor) of early-stage human aortic lesions were stained with Perls, DAB, or both (Perls + DAB). The Perls + DAB co-staining revealed that redox-active iron was detected more frequently in the FS (84\%) and FA (90\%) samples than in the HA $(22 \%)$ samples $(p<0.001$, Table 2$)$. Staining with Perls alone, which reveals the ferritin-associated FeIII with a low oxidative potential, was always negative in the tunica intimas of the HA samples and was positive in 9 and $39 \%$ of the FS and FA samples, respectively (Table 2). Similarly, staining with DAB alone, which reveals the pseudo-peroxidase activity from intact 


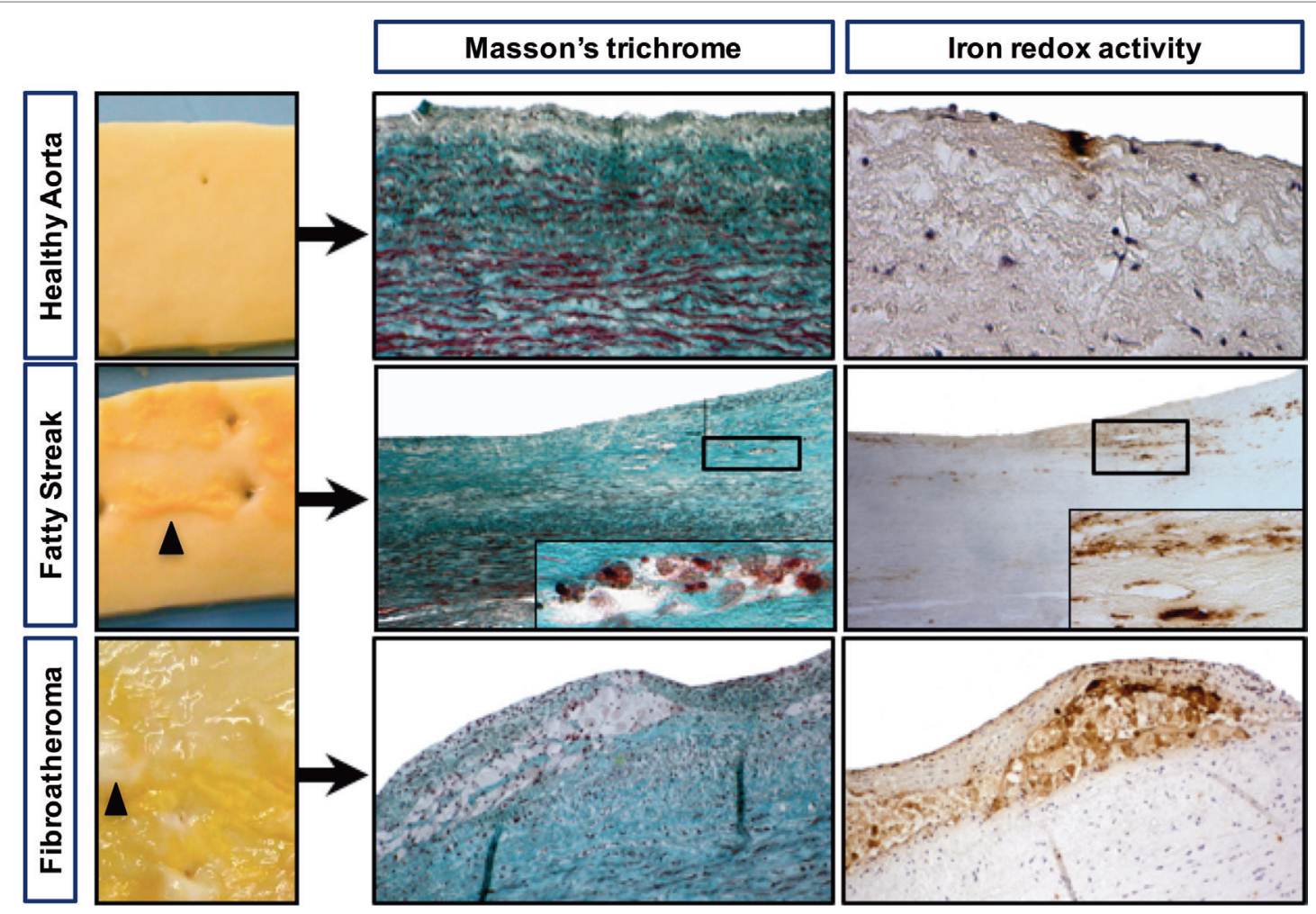

FIGURE 1 | Iron deposits are frequently detected in early-stage atheroma lesions. Macroscopic pictures of a healthy aorta (smooth luminal interface), a fatty streak with subendothelial yellow deposits (black arrowheads) and a fibroatheroma with yellow deposits covered with a white capsule, forming initial plaques (black arrowheads). Visualization of foam cells was accomplished by Masson's trichrome staining, and redox-active iron was visualized by Perls reaction followed by DiAminoBenzidin (DAB) polymerization (see materials and methods). Perls staining alone and DAB alone were strictly negative (not shown).

RBCs, was always negative in the tunica intimas and tunica medias from the HA samples, while $16 \%$ of the FS samples and $26 \%$ of the FA samples were positive (Table 2). Altogether, these data indicate that the redox-active iron is present as soon as FS and that iron-dependent oxidative stress is an early hallmark of plaque development.

To identify hemoglobin as a possible source of redox-active iron, we immunostained tissue sections with an anti-hemoglobin antibody. Hemoglobin was detected to a more or less extent on all pathological sections. In FS samples, hemoglobin was focally detected in the endothelium and in the vSMC-rich subendothelial intima (Figure 2A, a). In FA samples, the presence of hemoglobin was detected in the lipid core (Figure $\mathbf{2 A}, \mathrm{b}$ ). Frequently, $\mathrm{Hb}$ staining co-localized with $\mathrm{MYH}-11$ staining, corresponding to vSMCs. These qualitative results were confirmed by the measure of intimal heme content. We observed a trend for higher heme content in FS samples (Figure 2B). The tissue content of heme was significantly elevated in FA as compared to FS samples $(p<0.05)$ and HA $(p<0.01)$.

\section{Glycophorin A and Ceroids Can Be Detected in Early Aortic Lesions}

To accurately identify the presence of RBCs, we immunostained histological sections of FS and FA samples with an antibody against glycophorin A (Gly-A), which is a RBC membrane marker (Figure 3A, a-d). Gly-A immunostaining appeared as small granular points that could be differentiated from autofluorescence. Indeed, autofluorescent structures corresponding to ceroids (35) were often observed and co-localize with Gly-A (merged yellow fluorescence). We observed that these autofluorescent ceroids were present at a significantly lower frequency in the HA samples $(23 \% ; p<0.001)$ compared with those of the FS (71\%) and FA samples (94\%); not significantly different from FS samples (Table 1; Figure 3A, e,f). In addition, the ceroids predominantly appeared as round granules (Figure $\mathbf{3 A}$, e) in the HA and FS samples, whereas ceroid rings were more often observed in the FA samples (Figure 3A, f). As a lipofuscin pigment produced by oxidation, ceroids covalently link lipids to proteins and therefore could also be detected by ORO staining, despite the delipidation of the deparaffinized sections (Figure 3A, g,h).

Importantly, Gly-A was commonly associated with ceroids in the FS and FA samples. Gly-A was also found in the protein background of the lipid core and was associated with $\alpha$-actin and CD68 staining [Figure 3B, note that CD68 is a marker of phagolysosome activity (36) within phagocytes and can be expressed by vSMCs (37)].

We also found that Gly-A concentrations (Figure 4A) were significantly increased in the conditioned medium of 

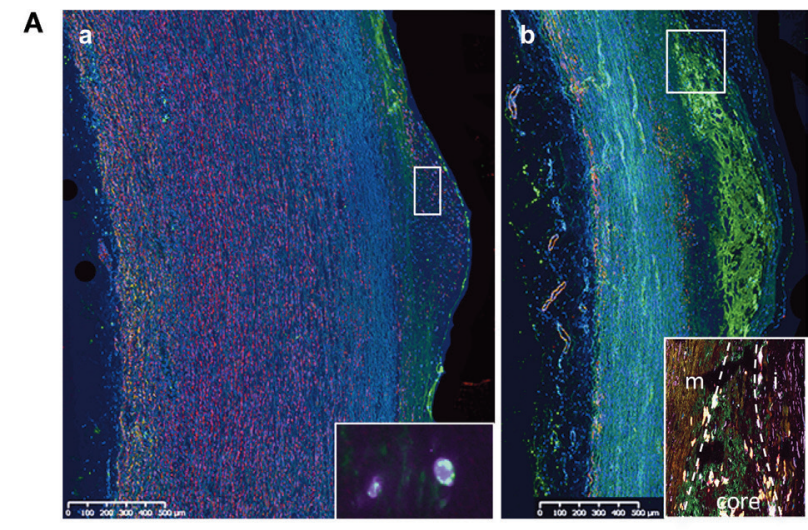

B

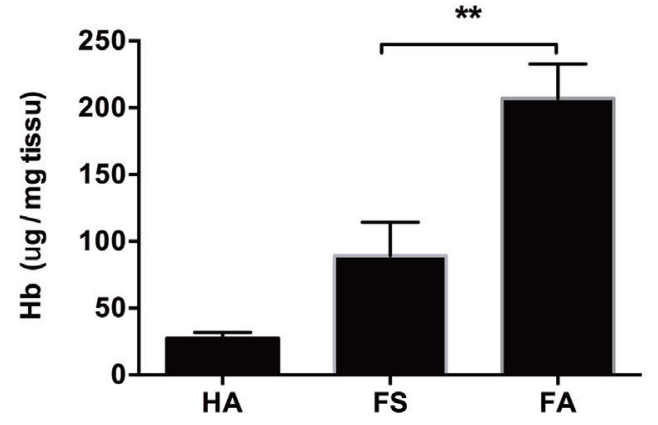

FIGURE 2 | Hemoglobin deposits in fatty streaks and fibro-atheroma lesions. Detection of $\mathrm{Hb}$ (green) and SMC myosin (pink) [(A), a] in the endothelium and subendothelial space of a fatty streak [inset: high magnification $(\times 100)$ showing the intracellular co-localization of myosin and $\mathrm{Hb}$ (merged yellow)], and $[(\mathbf{A}), \mathbf{b}]$ in in the lipid core (between the fibrocellular cap and the media) of a fibroatheroma lesion [inset: high magnification of the plaque shoulder ( $m$, media; i, intima; core shoulder) showing the presence of SMC myosin (pink) and hemoglobin (green), and their frequent localization (merged yellow)]. For negative control, there is no green autofluorescence in the lipid core (not shown). (B) Measurement of heme content in FS and FA tissue by formic acid reaction. ${ }^{* *} p<0.01$; Mann-Whitney's test.

the intimas of the FA samples compared with the conditioned medium of the intimas from the FS samples. Notably, for the HA samples, it was virtually impossible to separate and process the tunica intima, as indicated by "nd" in Figure 4 and the following figures. Gly-A concentrations were also significantly increased in the conditioned medium of the intimas from the FS and FA samples compared with those from the HA samples. Similarly, iron concentrations were significantly increased in the tunica intimas of the FA samples compared with those of the FS samples (Figure 4B). These results are consistent with the increased immunodetection of Gly-A in the lesions (Figure 3).

Together, these results indicate that augmented iron deposition and increased quantities of RBC-derived products in the arterial wall characterize both the initiation of FS formation and the progression toward FA and can be further associated with vSMC markers. Additionally, the presence of ceroids, which are insoluble polymers of oxidized lipids and proteins, and their frequent co-localization with Gly-A, indicate that RBC infiltration may promote local oxidative stress.

\section{Oxidative Stress Is Increased in Early Atheroma}

Thiobarbituric acid reactive substance (Figure 4C) concentrations were augmented in the conditioned medium prepared from the tunica intimas compared with that of the matched tunica medias. This difference was only statistically significant for the FA samples, and the concentrations tended to increase in the FS intimas. AOPPs concentrations (Figure 4D) were also significantly increased in the conditioned medium from the tunica intimas of the FA samples compared to media. Increased oxidation status was associated with significant overexpression of heme oxygenase-1 (HO-1) (Figure 4E) in the tunica intimas of the FS and FA samples compared with that of the corresponding tunica medias. The tunica medias of the FA samples had a higher oxidation status than did those of the FS samples.

\section{Iron Metabolism Pathway Gene Expression Is Altered in Early Human Atheroma}

The transcripts for NRAMP1 (a protein involved in iron transport between cell phagolysosomes and cytosol) and ferritin (a protein involved in iron storage) were significantly increased in the intimas of the FA samples (Figures 5A,B, respectively). Conversely, the transcription of ferroportin (a protein involved in iron export from cells) was decreased in the intimas of the FS (ns) and FA $(p<0.05)$ samples compared with that of the corresponding tunica media samples, suggesting iron retention within foam cells (Figure 5C).

In parallel, the levels of various transcripts for receptors potentially involved in phagocytosis, such as TIM-3, stabilin-1 (STAB1) and mannose receptor (MR) (CD206), were significantly increased in the tunica intimas of the FS and FA samples compared with that of their corresponding tunica medias (Figures 5D-F, respectively).

\section{Senescent RBCs Are Phagocytized by Primary vSMCs In Vitro}

We co-incubated either fresh or senescent RBCs (sRBCs), overexposing PS (2\% for fresh RBC, 98\% for senescent RBC) with human vSMCs in primary cultures. sRBC clearance by vSMCs was evaluated via kinetic counting of the remaining RBCs in the culture medium (Figure 6A). At 3 days of incubation, $76 \%$ of the sRBC were phagocytosed by vSMC. We also used a quantitative image stream approach (AMNIS), which showed an increased percentage of internalized sRBCs within vSMCs and an augmented coefficient of internalization after one day of incubation compared with the corresponding values obtained using fresh RBCs (Figure 6B).

Phagocytosis of sRBCs by human vSMCs was evaluated by Gly-A staining after 3 days of incubation (Figure 6C). We found that Gly-A signals drastically increased in vSMCs that were incubated with sRBCs compared with those that were incubated with fresh RBCs. Importantly, the Gly-A-positive cells were also positive for CD68.

Electron microscopy was used to confirm that vSMCs not only can engulf sRBCs but form and release exosomes, and retain lipophagic vacuoles, thereby endowing these cells with a 

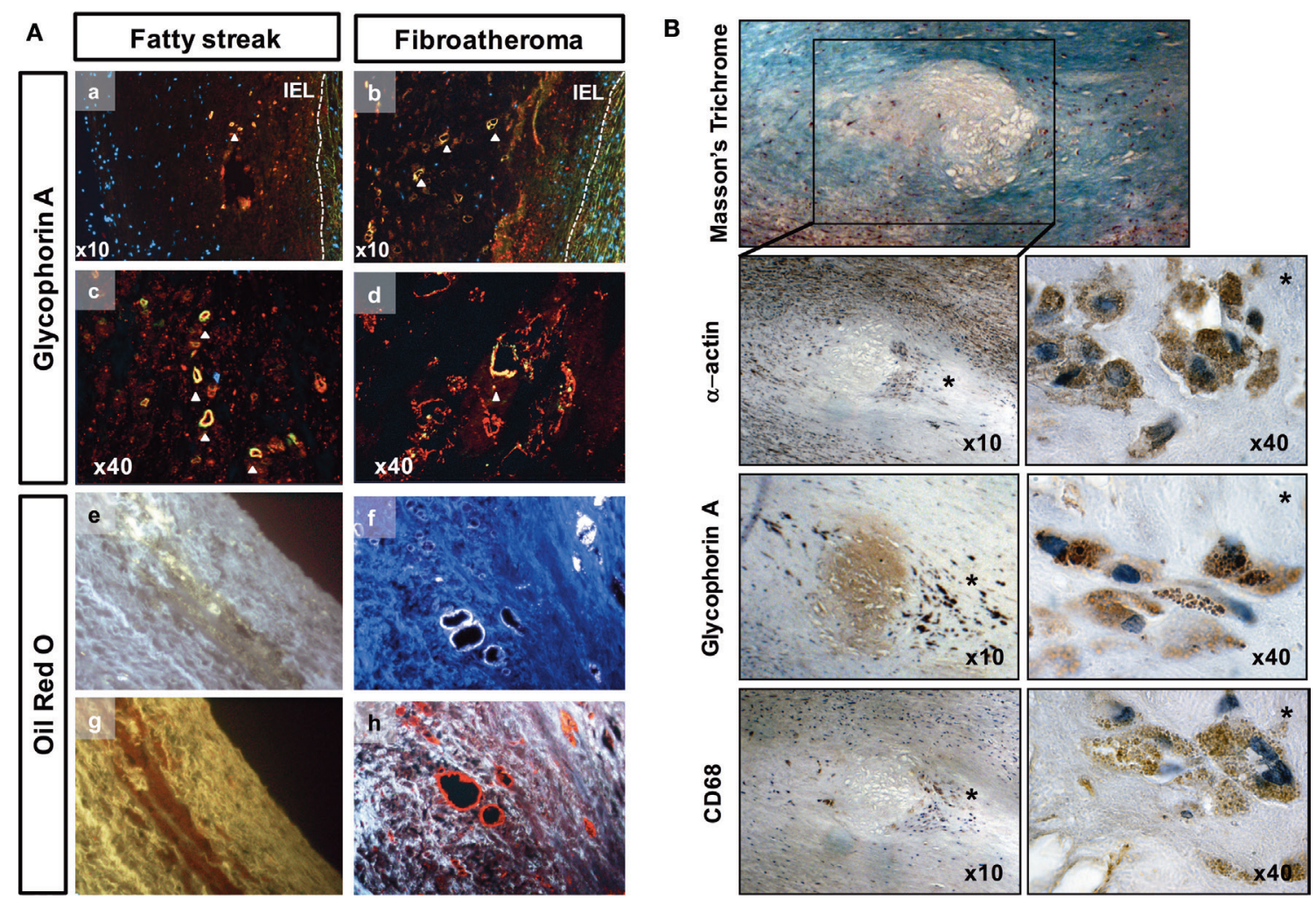

FIGURE 3 | Red blood cell phagocytosis in early-stage atheroma. (A) Detection of ceroids (autofluorescence) and glycophorin A (red) in the tunica intima of fatty streaks (FSs) and in lipid core of fibroatheromas (FAs) corresponding to high magnifications of pictures 2Aa (FS), b (FA), respectively. Granular and ring-shaped ceroids (merged yellow) co-localized ( $a-d)$ with glycophorin A immunostaining. Autofluorescent granular ceroids (e) and ceroid rings ( $f$ ) were detected in the tunica intimas of FS and Fas, respectively (white autofluorescence at a wavelength of $350 \mathrm{~nm}$ ). The ceroids were also characterized by their ability to bind ORO ( $\mathrm{g}$,h, red staining). IEL, internal elastic lamina. (B) Photographs of a lipid core surrounded by phagocytes in the shoulder region (*) of an initial plaque (Masson's trichrome). These phagocytes are $\alpha$-actin ${ }^{+}$, glycophorin A (Gly-A)+, and CD68+. Gly-A and CD68 specifically localized to cytosolic granules in the phagocytes (40x).

foam cell-like phenotype, but also can activate phagolysosomes, which degrade RBC structures (Figure 6D).

\section{Primary vSMCs Produce ROS after sRBC Phagocytosis}

We also observed that human cultured vSMCs engulfing sRBCs accumulated lipids and became ORO-positive (Figure 6E). In addition, phagocytosis of sRBCs by vSMCs resulted in oxidative stress, as shown by strong Perls + DAB staining of redox-active iron (Figure 6F) and a significant increase in reactive oxygen species (ROS) production (Figure 7A). The increased ROS production was dependent on NAPDH oxidase activity because the addition of apocynin, a NAPDH-specific inhibitor, strongly decreased ROS production (Figure 7A). We also observed that incubating vSMCs with $\mathrm{Hb}$ led to a dose-dependent increase in ROS production, which was partially blocked by desferoxiamine, providing evidence of the catalytic role of redox-active iron on endogenous oxidase activities (Figure 7B). In response, erythrophagy induced HO-1 and ferritin expression in vSMCs at both the mRNA (Figure 7C) and protein levels (Figure 7D). Importantly, $\mathrm{Hb}$ and HO-1 were concomitantly detected (Western blot) in vSMCs only when they were co-cultured with sRBCs (Figure 7D).

\section{Hypercholesterolemia in Rabbits Affects RBC Trafficking in the Aortic Intima}

We next tested whether experimental hypercholesterolemia in rabbits could reproduce some of our findings from human samples. As expected, after consuming a cholesterol-rich diet for 6 weeks, the rabbits developed aortic lipid-rich intimal atheroma. We also observed that diet-induced hypercholesterolemia led to a significant increase $(\times 3)$ in the proportion of circulating Annexin $\mathrm{V}^{+} \mathrm{RBCs}$ (Figure 8A). Strikingly, in the intimal lesions that developed in these hypercholesterolemic rabbits (Masson's trichrome), RBCs (GSL-B4+) were closely associated with strong RAM11 immunostaining and presence of redox-active iron (Perls + DAB staining; Figure 8B). These results indicate that FS lesions in hypercholesterolemic rabbits also contain iron deposits and infiltrated RBCs, in agreement with our observations of human FS and FA samples.

\section{An Overload of Circulating sRBCs Leads to Increased Arterial Iron Content in a Rat Model}

To experimentally assess RBC entry into the vascular wall and to determine whether the status of the endothelium is critical 

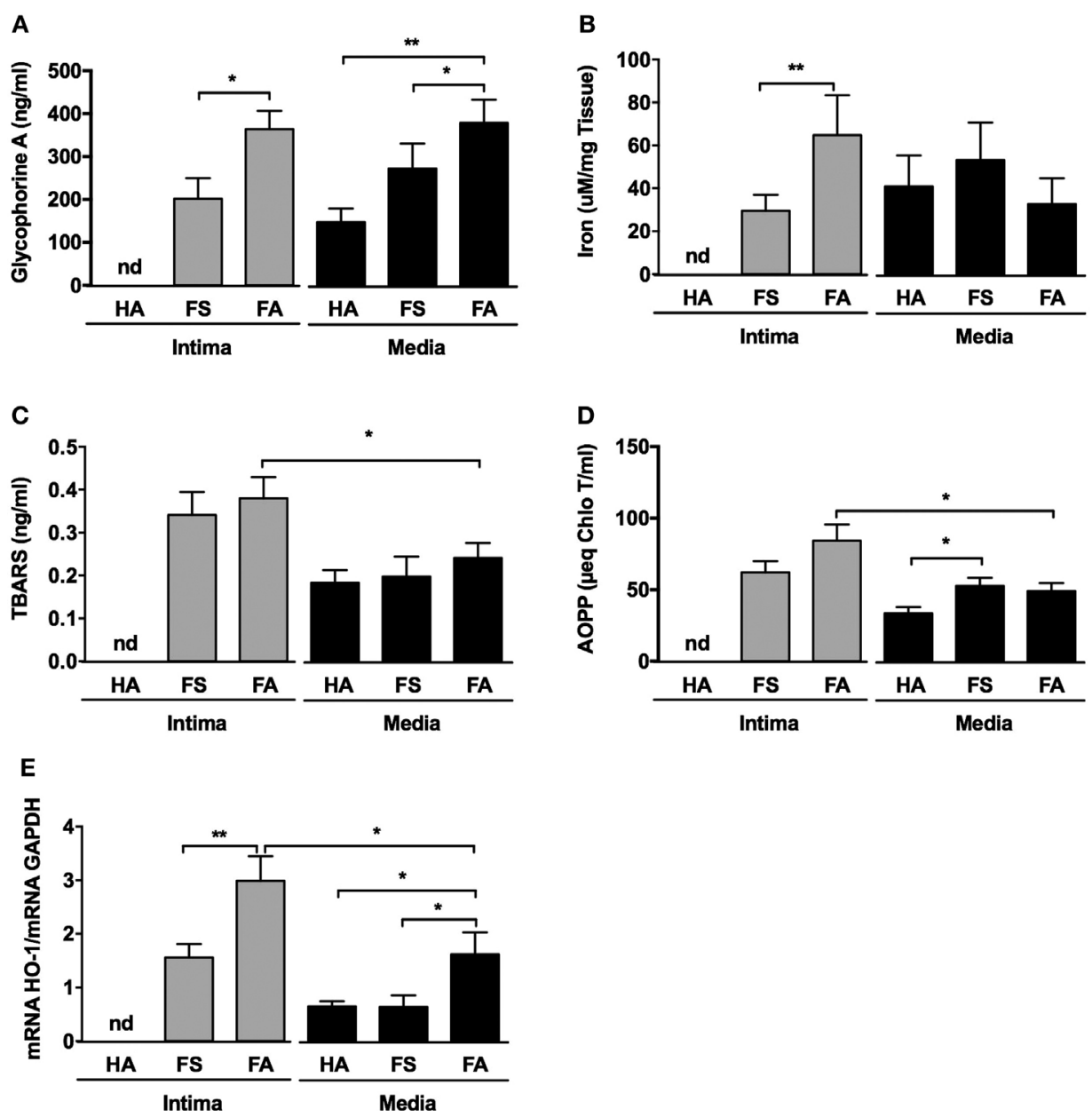

FIGURE 4 | Fatty streaks (FSs) and fibroatheromas (FAs) display high glycophorin A concentration, increased iron content, and augmented oxidative stress. (A) Glycophorin A concentration ( $n=10$ ) was determined in the conditioned media of healthy aortas (HAs), FSs, and FAs. (B) Iron content was evaluated in the corresponding tissues. (C,D) Thiobarbituric acid reactive substance (TBARS) $[n=10$, lipids, panel (C)] and advanced oxidation protein product (AOPP) $[n=10$, proteins, panel (D)] concentrations were determined in the same conditioned media described above. (E) The expression of Heme-Oxygenase-1 (HO-1) was evaluated in the intima and the tunica medias of healthy aortas (HA, $n=10)$ and from the tunica intimas $(n=10)$ and tunica medias $(n=10)$ of fatty streaks (FSs) and fibroatheromas (FAs). ${ }^{*} p<0.05,{ }^{* *} p<0.01$; Mann-Whitney's test.

for this process to occur, we submitted inbred Lewis rats to repeated injections of isogenic rat sRBCs. Two groups of rats were evaluated: in the first the aorta was left untouched, while the aorta was balloon-injured in the second. To further increase sRBC concentrations, a subset of the rats was splenectomized. The repeated injection of sRBCs significantly increased both average spleen weight (from $249.3 \pm 67 \mathrm{mg}$ in the controls to $462.4 \pm 140 \mathrm{mg}$ in the experimental group; $p=0.0002)$ and average spleen iron concentration $(314 \pm 33$ versus $469 \pm 40 \mu \mathrm{M} / \mathrm{mg}$ tissue; $p<0.01)$. Positive Perls, DAB, and Perls $+\mathrm{DAB}$ staining was found in the isolated aortas (Figure 9A). The para-aortic lymph nodes were also positive for Perls (Figure 9A). When sRBC injections were combined with ballooning and/or splenectomy, all of the above parameters significantly increased (Figure 9B). Factorial analysis of variance established that the injection of sRBCs was the most important factor underlying the increased aortic iron content (Figure 9C; $p<0.007$ ). However, ballooning and splenectomy also promoted iron accumulation in the aortic wall, even if their effects were more modest ( $p=0.015$ and $p=0.036$, respectively). There were no significant interactions between the three factors.

\section{DISCUSSION}

Our present observations confirm that FS and early FA are initiated in juxta intercostal/lumbar artery ostia in the dorsal part of the human aorta (4). Early FSs evolve into FAs, in which, intimal lipid deposits are recovered by the fibrocellular proliferation of vSMCs, while the lipid core of the initial plaque becomes acellular. Foam cells, characterized by intracellular accumulation of lipids, are observed during these early stages. Our present observations also confirm that intimal vSMCs have been pointed as key funders of foam cells and phagocytosis in the arterial wall (9).

In this context of human early atheroma, the roles of RBCs have not yet been fully recognized. Early studies focused mainly 

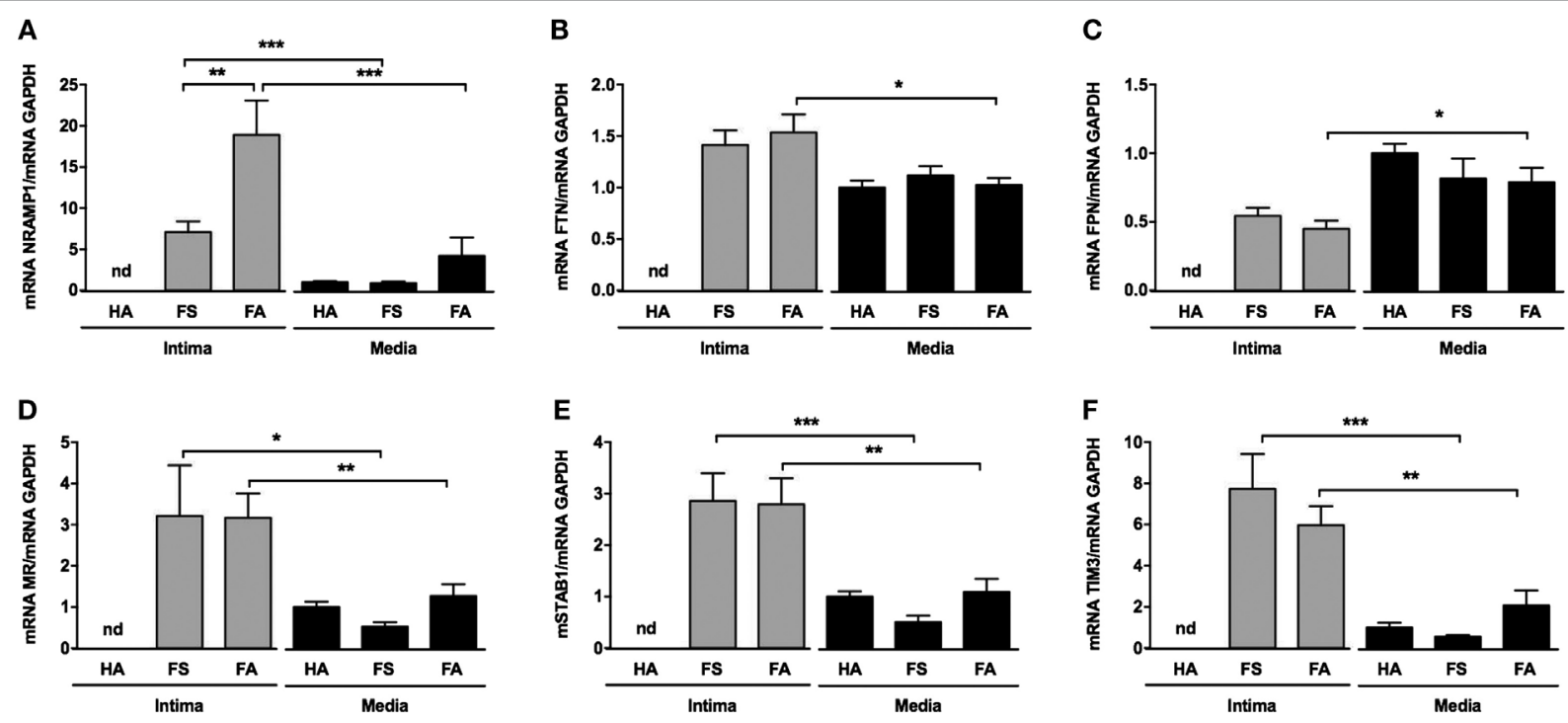

FIGURE 5 | Intraparietal red blood cells are associated with iron storage and expression of scavenger receptors. (A-C) mRNA levels of the following iron metabolism genes: NRAMP, ferritin, and ferroportin [(A-C), respectively]. (D-F) mRNA levels of the following phagocytosis genes: stabilin-1, mannose receptor (MR), and TIM3 [(D-F), respectively]. These quantifications were performed from mRNA isolated from the tunica medias of healthy aortas (HAs, $n=10)$ and from the tunica intimas $(n=10)$ and tunica medias $(n=10)$ of fatty streaks and fibroatheromas (FAs). ${ }^{*} p<0.05 ;{ }^{* *} p<0.01 ;{ }^{* * *} p<0.0001($ Mann-Whitney's test).

on the role of free hemoglobin on endothelial cells $(18,38)$ and of RBC in the late stages $(21,22)$ of atherothrombotic evolution. In the present study, we explored the possibility that $\mathrm{RBC}$ interactions with the wall and sRBC efferocytosis by vSMCs could be early priming events underlying lesion development in human arteries. Toward this end, we co-stained cells with Perls + DAB (34) to enhance detection redox-active iron, whereas Perls alone and DAB alone served as negative controls. The results provided evidence that this redox-active iron can catalyze endogenous oxidase activities, as shown by the DAB polymerization (32). Although no selection of tissue samples was performed, and despite the limited sample size, we nonetheless found a high percentage of tissue sections with FS or FA lesions in which redox-active iron was detected using this simple histochemical method. Redox-active iron was mainly detected in foam cells regardless of lesion stage. The presence of iron in these cells led them to adopt a granular, gray appearance, while simple lipid deposits appeared as translucent objects, as previously described by electron microscopy (39). Of note, intimal redox-active iron could be detected in apparently HAs, suggesting that iron acquisition by the intima, is a common occurrence and may represent an early event in the development of atheroma. In contrast, when evaluating ferric iron storage by staining with Perls alone, significantly less iron accumulation was found, demonstrating the low sensitivity of Perls staining for detecting redox active iron.

These data were confirmed by hemoglobin and heme detection in human FS and FA samples. Immunohistochemistry of hemoglobin (detecting the protein part of $\mathrm{Hb}$ ) showed that hemoglobin is detected at a high frequency in FS and FA samples. Importantly, hemoglobin detection could be associated with the detection of vSMC myosin, suggesting that the
vSMCs could be the effector and the target of intracellular $\mathrm{Hb}$ release. Heme assay showed an increase of the tissue heme content, which followed the progression of the pathology. In this context, $\mathrm{Hb}$ and heme appear as molecular intermediate metabolites between sRBC efferocytosis and intracellular iron accumulation.

Strikingly, the frequency of redox-active iron staining was similar to the frequency with which we detected ceroid lipofuscin. Ceroids are autofluorescent, insoluble polymers of oxidized lipids and proteins. They can form intracellularly (40), appearing as punctate, small particles of gruel, depending on the presence of redox-active iron and phagolysosome activity (41), or they can form extracellularly, appearing as rings. Our observations that ceroids co-localized with redox-active iron deposits and myosin within the cell and further in the extracellular compartment $(42,43)$ might indicate that ceroids primarily could form within the intracellular compartment of vSMC. Importantly, results from Raman and fluorescence spectroscopy have been recently used to demonstrate the presence of $\mathrm{Hb}$ and oxidase within ceroids (44).

To determine whether intimal redox-active iron is derived from $\mathrm{RBC}$ or from free $\mathrm{Hb}$, we stained tissue sections with an anti-Gly-A antibody to visualize the presence of RBC membranes (21). In the FS and FA samples, iron and Gly-A were often co-localized. RBC membranes provide an important source of unesterified cholesterol in advanced hemorrhagic plaques (21). The presence of Gly-A in the FS and FA samples strongly suggests that cholesterol derived from RBC membranes is also a significant contributor of engulfed and extracellular cholesterol in early lesions, as has shown for advanced lesions $(20,21)$. Moreover, in atheromatous tissues, we observed that intracellular Gly-A could co-localize with $\mathrm{CD}^{+} 8^{+}$phagolysosomal structures 

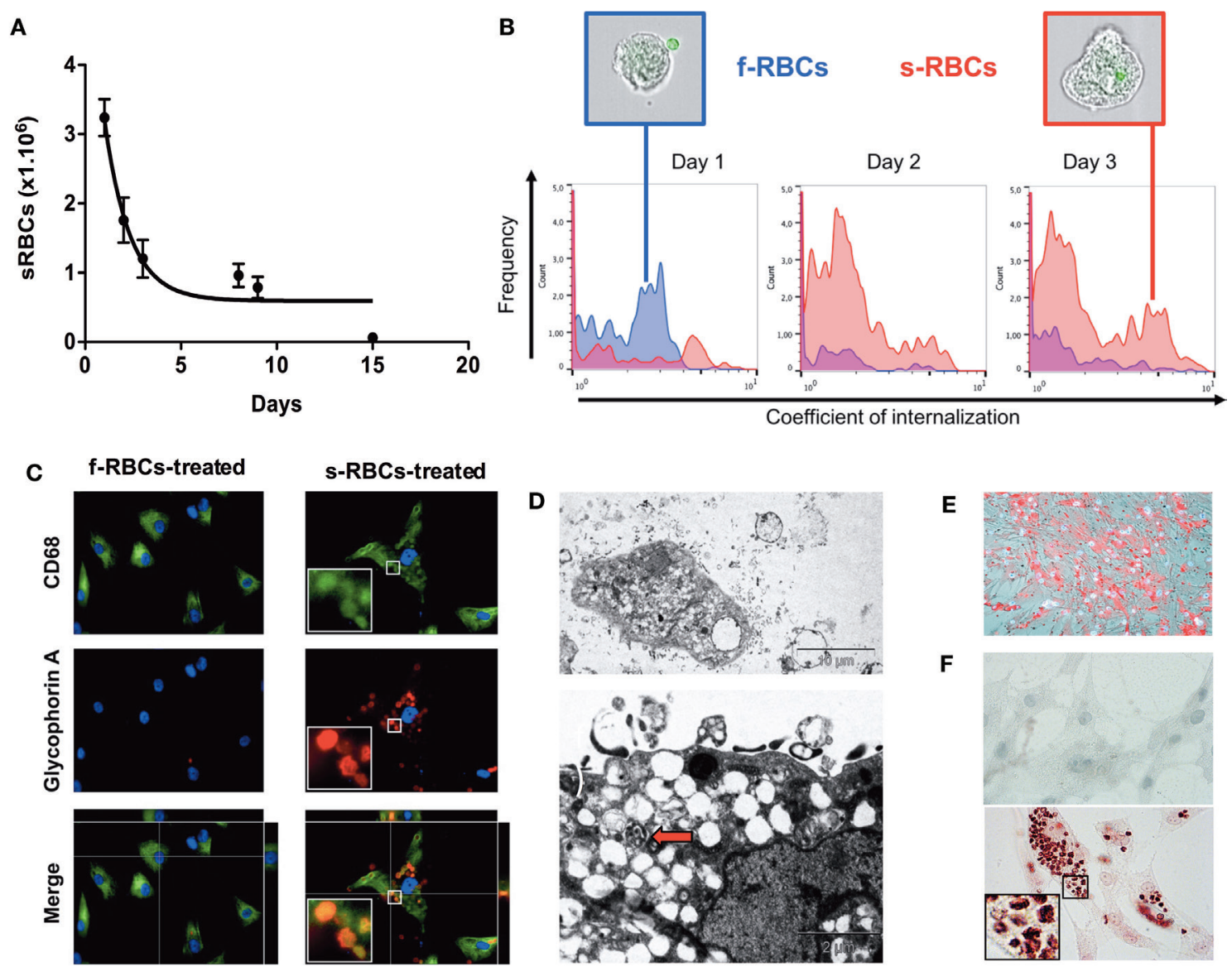

$\mathbf{F}$

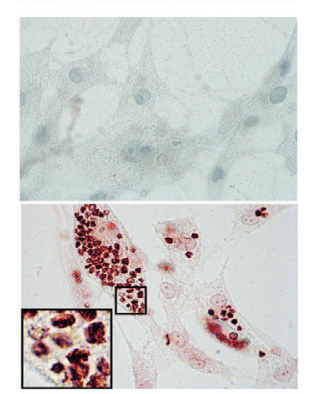

FIGURE 6 | Vascular smooth muscle cells (vSMCs) phagocytose senescent red blood cells (sRBCs) in vitro. (A) Time course of the diminution of sRBC in the culture medium of VSMCs. The purity of the culture was obtained by dissecting the media form the adventitia. The vSMC nature was validated by $\alpha$-actin and transgelin staining (not shown) (B) Internalization coefficient of RBCs in resuspended vSMCs at days 1,2 , and 3 ( $n=5$ for each time point) calculated by the IDEAS ${ }^{\circledR}$ software (Amnis). vSMCs with a low coefficient are plotted on left side of each histogram, as observed with fresh RBCs (f-RBCs; blue curves; note the presence of a CFSE + RBC outside of the VSMC in the representative image in the blue-colored frame). vSMCs with a high internalization coefficient will be displayed on the right side, as illustrated with vSMCs co-cultured with senescent RBCs (sRBCs; red curves; an internalized CFSE + RBC can be detected inside the vSMC shown in the red-colored frame). (C) Glycophorin A and CD68 staining of VSMCs incubated with fresh RBCs or with senescent RBCs for 3 days. (D) Electron microscopy of vSMCs that were co-cultured with RBCs. Note that VSMCs internalized SRBCs, formed and released exosomes, retained lipophagic translucent vacuoles that endowed these cells with a foam cell-like phenotype, and displayed phagolysosomes (red arrow). (E) Oil Red O staining of membrane cholesterol from RBCs incorporated by vSMCs. (F) Perls + DAB staining of redox-active iron in vSMCs incubated with fresh RBCs (higher panel, negative) or with senescent RBCs (lower panel, positive) for 3 days.

and with intracellular or extracellular ceroids in foam cells and lipid core. We also observed the presence of glycophorin A in ceroids providing evidence that one part of the oxidized lipids came from RBC membranes in ceroids. The co-localization of redox-active iron, lipids, and Gly-A and the involvement of the phagolysosome in ceroid formation suggest that these pathological structures are closely associated with senescent erythrocyte efferocytosis in early atheroma.

We also observed greater release of TBARS and AOPPs, markers of lipid and protein oxidation, respectively, from the intima of early lesions than from the corresponding tunica medias, suggesting that the oxidation process initiates in the intima. In direct response to heme, activation of NF-KB (38), induction of ferritin (45), and heme-oxygenase-1 (HO-1) (46) partially protect endothelial cells from oxidative injury. We detected HO-1 overexpression in our FS and FA samples, which could act as a compensatory mechanism, explaining the limited increases in AOPPs and TBARS concentrations observed in cell-rich FSs. This compensatory mechanism would be more limited in the lipid core because of the loss of vSMCs. In parallel, the expression levels of mRNA encoding proteins involved in intracellular iron metabolism were also modified. In particular, NRAMP1, which acts to transport divalent iron across the phagolysosomal membrane (47), and ferritin (48), an ubiquitous intracellular protein that stores and releases iron (49), were upregulated. Conversely, ferroportin-1, a transmembrane iron exporter (50), was downregulated. Taken together, these observations suggest that iron storage is increased in the atherosclerotic vessel wall. Moreover, several proteins that promote phagocytosis after the recognition of exposed PS were also upregulated in FS and FA 

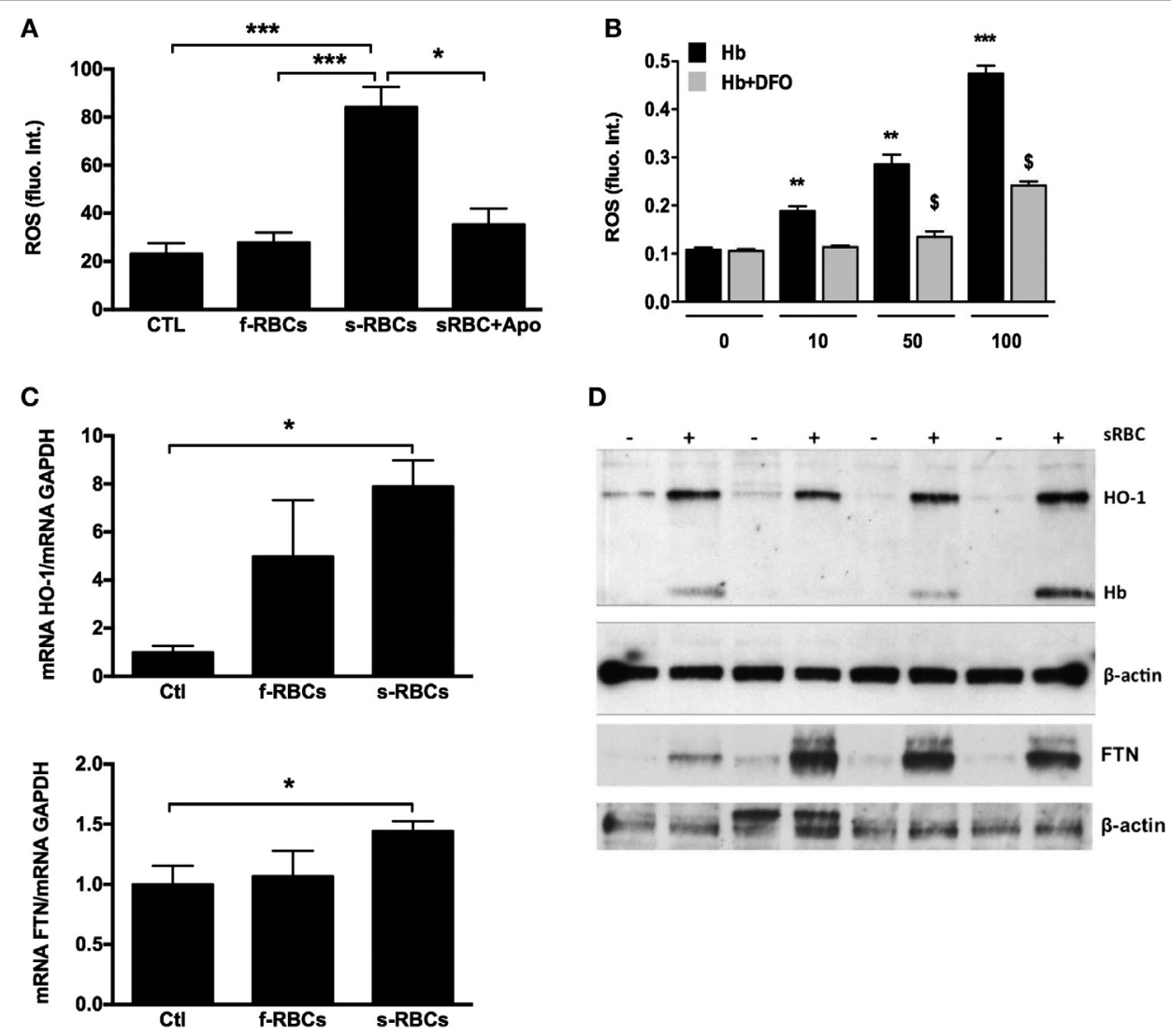

FIGURE 7 | Senescent red blood cell (SRBC) phagocytosis induces reactive oxygen species (ROS) production and heme oxygenase-1 (HO-1) and ferritin expression. (A) Vascular smooth muscle cells (vSMCs) were incubated with fresh or RBCs ( $1 \times 10^{6}$ cells) for 3 days ( $\left.n=6\right)$, and ROS production was assessed by fluorometry. (B) vSMCs were incubated with increasing concentrations of hemoglobin $(0-100 \mu \mathrm{M}) \pm$ desferoxiamine $(20 \mu \mathrm{M})$ for $2 \mathrm{~h}(\mathrm{n}=6)$, and ROS production was assessed as in (A). (C,D) HO-1 and ferritin expression in vSMCs after 3 days of incubation with senescent RBCs $(n=4)$ were determined by RT-PCR (C) and Western blot (D). Intracellular hemoglobin content (D) was determined by Western blot.

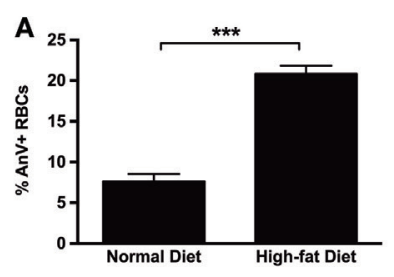

B

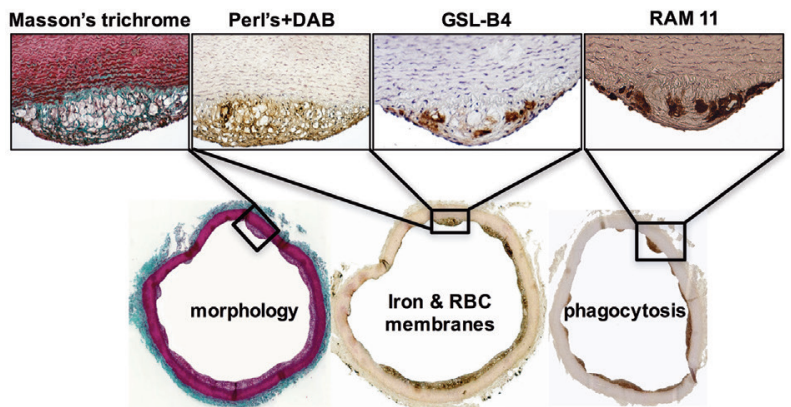

FIGURE 8 | High-cholesterol diet induces red blood cell accumulation in rabbit foam cells. (A) After consuming a hypercholesterolemic diet for 3 months, rabbits showed significantly increased percentages of circulating Annexin $V^{+} R B C s$ ( ${ }^{\star \star *} p<0.001$; Mann-Whitney's test). (B) The consumption of a hypercholesterolemic diet was associated with the development of focal subendothelial lesions characterized by the predominance of foam cells, as shown by Masson's trichrome staining. These foam cells were redox-active iron-positive based on Perls + DiAminoBenzidin (DAB) staining (Perls alone and DAB alone were strictly negative, not shown). They were also recognized by a rabbit RBC membrane marker (GSL-B4 lectin) and were found to express a phagocyte marker (RAM11).

samples, including TIM3, which possesses a domain allowing for highly specific recognition of exposed PS (51); STAB1, a scavenger receptor that interacts with numerous ligands, including PS (52); and MR, also known as CD206, a C-type lectin primarily present on the surfaces of phagocytes that can recognize various cell-surface saccharide structures (53). Together, the above data strongly suggest that the trafficking of RBCs across the intima is associated with increased iron storage and an increased capacity 

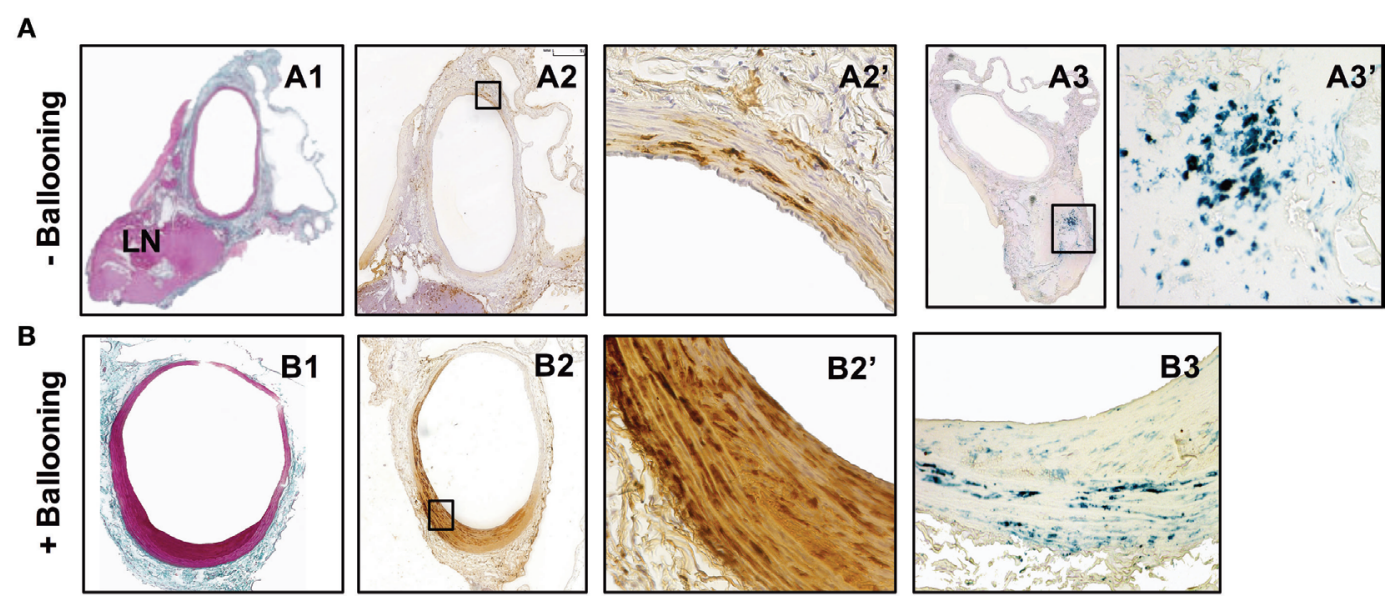

C

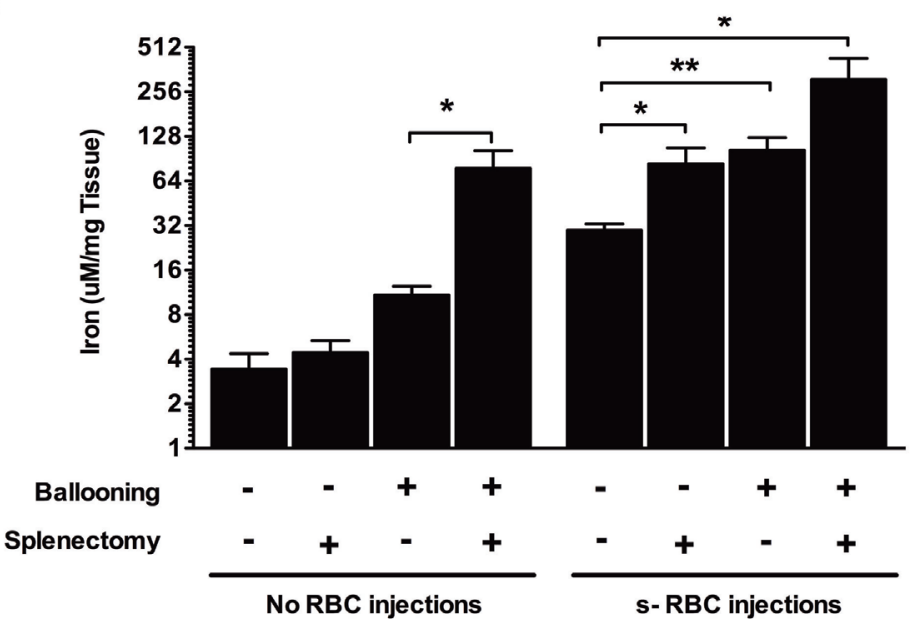

FIGURE 9 | Balloon injury promotes red blood cell phagocytosis. Rats were either subjected to intravenous injections of senescent RBCs once a week for 2 weeks or were not. Subsequently, a subset of the rats was submitted to ballooning of the abdominal aorta. [line (A)] Aorta from a rat injected with senescent RBCs without ballooning. Masson's trichrome staining (A1) of the aorta associated with an adjacent lymph node. Areas positive for Perls + DiAminoBenzidin (DAB) stainig (A2, A2') were observed throughout the media, indicating the presence of redox-active iron (negative for Perls or DAB alone, not shown). Perls staining (A3, A3') revealed a continuum of iron transport and metabolism between the arterial wall and the local LN. (B) Aorta of a rat injected with senescent RBCs after ballooning. Masson's trichrome staining (B1) showing intimal proliferation. Perls + DAB staining (B2, B2') indicating the presence of redox-active iron in the intima and media. Perls alone staining (B3), showing an accumulation of ferric iron (sequestered form potentially linked to ferritin) in the arterial wall. (C) Concentrations (logarithmic scale) of iron in the aortas of different groups of rats following senescent RBC injections, ballooning and/or splenectomy ( $n=10$ for each group), as determined by

spectrophotometry. ${ }^{*} p<0.05$ and ${ }^{* *} p<0.01$; Mann-Whitney's test.

to recognize and uptake senescent cells. These events could favor the formation of foam cells and the release of redox-active iron from $\mathrm{RBC} /$ heme, which could be instrumental in inducing intimal oxidative processes.

To directly test this hypothesis, we incubated senescent or fresh RBCs with primary cultures of vSMCs. vSMCs were chosen because they are the main resident stromal cells of the arterial wall and are predominantly involved in FSs and in the fibrocellular caps of FAs. vSMCs are highly plastic $(13,54)$; they can acquire a $\mathrm{CD} 8^{+}$macrophage-like phenotype $(8,10-12)$ and can phagocytose sRBCs (23). The transient presence of Gly-A and hemoglobin, the retention of redox-active iron, the formation of lipid vacuoles, and the intense activation of the phagolysosome and exocytosis provide evidences of erythrophagy by vSMCs. As in tissues, sRBC efferocytose by vSMCs promoted oxidation by catalyzing oxidase activity and induced HO-1 and ferritin expression as factors limiting oxidative injury $(45,46)$.

Finally, we explored the relevance of the above mechanism in vivo in experimental models. The induction of atheroma in rabbits via the provision of a cholesterol-rich diet is a wellestablished model that can be characterized by the formation of a proliferative neo-intima that is particularly rich in lipid-laden foam cells and originates, at least in part, from resident vSMCs (55). In an interesting study, Nakayama and colleagues explored HO- 1 and bilirubin expression in a high-fat-diet rabbit model (56). They found that intimal foam cells express and accumulate HO- 1 and bilirubin (a product of heme degradation), suggesting 
that these cells metabolized heme. However, the above studies did not address the origin of the heme. In parallel, Pang and colleagues reported ferritin overexpression in the aortas of hypercholesterolemic rabbits (48), and Lee et al. reported the presence of iron (based on Perls + DAB histochemistry and electron microscopy results) in rabbit foam cells (57). In the present study, we identified RBCs as a potential source of heme because the intimal foam cells in our hypercholesterolemic rabbits contained redox-active iron, showed phagolysosome activation (RAM-11) and stained positive for RBC cell-surface saccharides (isolectin B4). Although we recognize the poor specificity of this marker, to the best of our knowledge, it is the only RBC membrane marker available for use in rabbits.

Hypercholesterolemia is known to impact RBC membrane function by chelating chemokines through the Duffy antigen, promoting erythrophagy through PS exposure, and augmenting interactions between endothelial cells and leukocytes in rabbits (30), mice (58), and humans (59). Accordingly, we found an increased percentage of sRBCs in our hypercholesterolemic rabbits. Therefore, by enhancing the proportion of sRBCs, a hypercholesterolemic status can potentially increase RBC interactions with the arterial wall.

Therefore, we next tested whether RBCs must be senescent to interact with cells in the vascular wall and whether the vascular endothelium must be intact to support these interactions. For this purpose, we developed a model of repeated intravenous injections of sRBCs in rats. Aortic endothelium was either left untouched or mechanically removed. In addition, a subset of the rats was subjected to splenectomy. Splenectomy was expected to significantly reduce the clearance of circulating sRBCs. It is important to note that rats are particularly resistant to atheroma. Strikingly, we observed that intravenous injection of sRBCs led to their convection across the arterial wall and to iron retention within the aorta. This iron retention was significantly enhanced by ballooning and/or splenectomy. These data strongly suggest that RBC trafficking across the aortic wall could be a pathogenic event that increases the iron content within the wall. The mechanism by which RBCs can traffic across the vessel wall remains to be clarified. Our data indicate that the integrity of the endothelium offers only relative protection to the arterial wall. While we have shown that erythrophagy by vSMCs can indeed lead to the transformation of vSMC into foam cells and increase their oxidative potential. We recognize that other cells might display the same capacities. In addition, our study does not exclude the possibility that the release and endocytosis of free $\mathrm{Hb}$ can occur independently of erythrophagy. Nevertheless, we washed our senescent RBCs in order to focus on RBC rather than to free hemoglobin. Results of our experimental design in rats are similar to the results published by Baek et al. (19), showing that transfusion of long-term stored RBCs (likely similar to the sRBCs that we used in our experiments), sensitive to spontaneous hemolysis, was highly toxic for the arterial wall in guinea-pigs. In order to minimize the direct toxic effect of $\mathrm{Hb}$, fresh and $\mathrm{sRBC}$ were washed before use in our study.

The clinical significance of our findings is multifaceted. Indeed, the major risk factors (i.e., hypercholesterolemia, smoking, diabetes, aging) can alter the biology of RBCs. We have observed that hypercholesterolemia induced a three-fold increase in the proportion of senescent RBCs in rabbits. This has been also reported from the use of a high-fat-diet mouse model (58). Diabetes as well as smoking (60) was also reported to increase the proportion of sRBCs in human. Therefore, our study indicates the need for a thorough evaluation of $\mathrm{RBC}$ status in atherothrombosis clinical studies to test whether risk factors with unknown pathophysiological mechanisms could act through an effect on RBCs, to evaluate whether current drugs act on RBCs, and to design new treatments aimed at preventing RBC dysfunction.

Most atherothrombosis studies focus on leukocytes. This study is unique because it focused on erythrocytes. In evaluating human tissues, cell cultures, and experimental animal models, we have demonstrated that RBCs can enter the arterial wall during the earliest stages of atheroma and are phagocytosed by vSMC. This entry serves as a pathogenic mechanism and is enhanced by hypercholesterolemia, endothelial injury, and RBC senescence. Because RBCs carry cholesterol and phospholipids in their membranes and are an iron/heme cargo, their infiltration and phagocytosis by vSMCs appear to be instrumental processes underlying the formation of foam cells, the genesis of intraparietal oxidation and finally the anti-oxidant response of vSMC. Therefore, this study unravels a previously unforeseen pathophysiological mechanism of plaque formation where erythrocyte angiophagy by vSMCs is a key developmental trigger.

\section{ETHICS STATEMENT}

Human aortas were consecutively collected from deceased organ donors from 2010 to 2013 under the authorization of the French Biomedicine Agency (PFS 09-007). All experimental and animal care procedures complied with the principles formulated by the National Society for Medical Research (animal facility agreement: No. B75-18-03). This study was performed under the advice of our institution's animal ethical committee (authorization no. 75-101) and complied with all guidelines for animal experimentation set forth by the French Ministry of Agriculture.

\section{AUTHOR CONTRIBUTIONS}

Study design: SD, RB, KG, AN, and J-BM. Acquisition of the data: SD, RB, BH-T-N, JL, ZT, VO, CD, MM, LL, and KG. Analysis and interpretation of the data: SD, RB, BH-T-N, AN, and J-BM. Manuscript preparation: SD, BH-T-N, KG, AN, and J-BM.

\section{ACKNOWLEDGMENTS}

We would like to acknowledge the ImagoSeine core facility of the Institut Jacques Monod, a member of the IBiSA and FranceBioImaging (ANR-10-INBS-04) infrastructures. This study was supported by the French Foundation for Medical Research (FRM) and French Foundation (FF). 


\section{REFERENCES}

1. Tabas I, Williams KJ, Boren J. Subendothelial lipoprotein retention as the initiating process in atherosclerosis: update and therapeutic implications. Circulation (2007) 116:1832-44. doi:10.1161/CIRCULATIONAHA.106. 676890

2. Biasetti J, Spazzini PG, Hedin U, Gasser TC. Synergy between shear-induced migration and secondary flows on red blood cells transport in arteries: considerations on oxygen transport. JR Soc Interface (2014) 11:20140403. doi:10.1098/rsif.2014.0403

3. Tarbell JM. Mass transport in arteries and the localization of atherosclerosis. Annu Rev Biomed Eng (2003) 5:79-118. doi:10.1146/annurev. bioeng.5.040202.121529

4. Mohamied Y, Rowland EM, Bailey EL, Sherwin SJ, Schwartz MA, Weinberg PD. Change of direction in the biomechanics of atherosclerosis. Ann Biomed Eng (2015) 43:16-25. doi:10.1007/s10439-014-1095-4

5. White SJ, Newby AC, Johnson TW. Endothelial erosion of plaques as a substrate for coronary thrombosis. Thromb Haemost (2016) 115:509-19. doi:10.1160/TH15-09-0765

6. Yla-Herttuala S, Palinski W, Rosenfeld ME, Parthasarathy S, Carew TE, Butler S, et al. Evidence for the presence of oxidatively modified low density lipoprotein in atherosclerotic lesions of rabbit and man. J Clin Invest (1989) 84:1086-95. doi:10.1172/JCI114271

7. Geer JC, Catsulis C, Mcgill HC Jr, Stron JP. Fine structure of the baboon aortic fatty streak. Am J Pathol (1968) 52:265-86.

8. Allahverdian S, Chehroudi AC, Mcmanus BM, Abraham T, Francis GA. Contribution of intimal smooth muscle cells to cholesterol accumulation and macrophage-like cells in human atherosclerosis. Circulation (2014) 129:1551-9. doi:10.1161/CIRCULATIONAHA.113.005015

9. Ho-Tin-Noe B, Vo S, Bayles R, Ferriere S, Ladjal H, Toumi S, et al. Cholesterol crystallization in human atherosclerosis is triggered in smooth muscle cells during the transition from fatty streak to fibroatheroma. J Pathol (2017) 241:671-82.

10. Feil S, Fehrenbacher B, Lukowski R, Essmann F, Schulze-Osthoff K, Schaller $\mathrm{M}$, et al. Transdifferentiation of vascular smooth muscle cells to macrophage-like cells during atherogenesis. Circ Res (2014) 115:662-7. doi:10.1161/ CIRCRESAHA.115.304634

11. Shankman LS, Gomez D, Cherepanova OA, Salmon M, Alencar GF, Haskins RM, et al. KLF4-dependent phenotypic modulation of smooth muscle cells has a key role in atherosclerotic plaque pathogenesis. Nat Med (2015) 21:628-37. doi:10.1038/nm.3866

12. Chappell J, Harman JL, Narasimhan VM, Yu H, Foote K, Simons BD, et al. Extensive proliferation of a subset of differentiated, yet plastic, medial vascular smooth muscle cells contributes to neointimal formation in mouse injury and atherosclerosis models. Circ Res (2016) 119:1313-23. doi:10.1161/CIRCRESAHA.116.309799

13. Lacolley P, Regnault V, Nicoletti A, Li Z, Michel JB. The vascular smooth muscle cell in arterial pathology: a cell that can take on multiple roles. Cardiovasc Res (2012) 95:194-204. doi:10.1093/cvr/cvs135

14. Ross R. Atherosclerosis - an inflammatory disease. N Engl J Med (1999) 340:115-26. doi:10.1056/NEJM199901143400207

15. Massberg S, Brand K, Gruner S, Page S, Muller E, Muller I, et al. A critical role of platelet adhesion in the initiation of atherosclerotic lesion formation. J Exp Med (2002) 196:887-96. doi:10.1084/jem.20012044

16. Jeney V, Balla G, Balla J. Red blood cell, hemoglobin and heme in the progression of atherosclerosis. Front Physiol (2014) 5:379. doi:10.3389/ fphys.2014.00379

17. Balla G, Vercellotti GM, Muller-Eberhard U, Eaton J, Jacob HS. Exposure of endothelial cells to free heme potentiates damage mediated by granulocytes and toxic oxygen species. Lab Invest (1991) 64:648-55.

18. Balla G, Jacob HS, Eaton JW, Belcher JD, Vercellotti GM. Hemin: a possible physiological mediator of low density lipoprotein oxidation and endothelial injury. Arterioscler Thromb (1991) 11:1700-11. doi:10.1161/01.ATV. 11.6.1700

19. Baek JH, D’agnillo F, Vallelian F, Pereira CP, Williams MC, Jia Y, et al. Hemoglobin-driven pathophysiology is an in vivo consequence of the red blood cell storage lesion that can be attenuated in guinea pigs by haptoglobin therapy. J Clin Invest (2012) 122:1444-58. doi:10.1172/JCI59770
20. Arbustini E, Morbini P, D’armini AM, Repetto A, Minzioni G, Piovella F, et al. Plaque composition in plexogenic and thromboembolic pulmonary hypertension: the critical role of thrombotic material in pultaceous core formation. Heart (2002) 88:177-82. doi:10.1136/heart.88.2.177

21. Kolodgie FD, Gold HK, Burke AP, Fowler DR, Kruth HS, Weber DK, et al. Intraplaque hemorrhage and progression of coronary atheroma. $\mathrm{N} \mathrm{Engl}$ J Med (2003) 349:2316-25. doi:10.1056/NEJMoa035655

22. Nagy E, Eaton JW, Jeney V, Soares MP, Varga Z, Galajda Z, et al. Red cells, hemoglobin, heme, iron, and atherogenesis. Arterioscler Thromb Vasc Biol (2010) 30:1347-53. doi:10.1161/ATVBAHA.110.206433

23. Kolb S, Vranckx R, Huisse MG, Michel JB, Meilhac O. The phosphatidylserine receptor mediates phagocytosis by vascular smooth muscle cells. JPathol (2007) 212:249-59. doi:10.1002/path.2190

24. Llorente-Cortes V, Otero-Vinas M, Camino-Lopez S, Costales P, Badimon L. Cholesteryl esters of aggregated LDL are internalized by selective uptake in human vascular smooth muscle cells. Arterioscler Thromb Vasc Biol (2006) 26:117-23. doi:10.1161/01.ATV.0000193618.32611.8b

25. Bennett MR, Gibson DF, Schwartz SM, Tait JF. Binding and phagocytosis of apoptotic vascular smooth muscle cells is mediated in part by exposure of phosphatidylserine. Circ Res (1995) 77:1136-42. doi:10.1161/01. RES.77.6.1136

26. Stary HC, Chandler AB, Glagov S, Guyton JR, Insull W Jr, Rosenfeld ME, et al. A definition of initial, fatty streak, and intermediate lesions of atherosclerosis. A report from the Committee on Vascular Lesions of the Council on Arteriosclerosis, American Heart Association. Circulation (1994) 89:2462-78. doi:10.1161/01.CIR.89.5.2462

27. Bentzon JF, Otsuka F, Virmani R, Falk E. Mechanisms of plaque formation and rupture. Circ Res (2014) 114:1852-66. doi:10.1161/CIRCRESAHA.114. 302721

28. Delbosc S, Diallo D, Dejouvencel T, Lamiral Z, Louedec L, MartinVentura JL, et al. Impaired high-density lipoprotein anti-oxidant capacity in human abdominal aortic aneurysm. Cardiovasc Res (2013) 100:307-15. doi:10.1093/cvr/cvt194

29. Hyafil F, Laissy JP, Mazighi M, Tchetche D, Louedec L, AdleBiassette $\mathrm{H}$, et al. Ferumoxtran-10-enhanced MRI of the hypercholesterolemic rabbit aorta: relationship between signal loss and macrophage infiltration. Arterioscler Thromb Vasc Biol (2006) 26:176-81. doi:10.1161/01. ATV.0000194098.82677.57

30. Tziakas D, Chalikias G, Kapelouzou A, Tentes I, Schafer K, Karayannakos P, et al. Erythrocyte membrane cholesterol and lipid core growth in a rabbit model of atherosclerosis: modulatory effects of rosuvastatin. Int J Cardiol (2013) 170:173-81. doi:10.1016/j.ijcard.2013.10.070

31. Clowes AW, Karnowsky MJ. Suppression by heparin of smooth muscle cell proliferation in injured arteries. Nature (1977) 265:625-6. doi:10.1038/ $265625 \mathrm{a} 0$

32. Seligman AM, Karnovsky MJ, Wasserkrug HL, Hanker JS. Nondroplet ultrastructural demonstration of cytochrome oxidase activity with a polymerizing osmiophilic reagent, diaminobenzidine (DAB). J Cell Biol (1968) 38:1-14. doi:10.1083/jcb.38.1.1

33. Nguyen-Legros J, Bizot J, Bolesse M, Pulicani JP. ["Diaminobenzidine black" as a new histochemical demonstration of exogenous iron (author's transl)]. Histochemistry (1980) 66:239-44. doi:10.1007/BF00495737

34. Meguro R, Asano Y, Odagiri S, Li C, Iwatsuki H, Shoumura K. Nonhemeiron histochemistry for light and electron microscopy: a historical, theoretical and technical review. Arch Histol Cytol (2007) 70:1-19. doi:10.1679/ aohc.70.1

35. Mitchinson MJ, Hothersall DC, Brooks PN, De Burbure CY. The distribution of ceroid in human atherosclerosis. J Pathol (1985) 145:177-83. doi:10.1002/ path. 1711450205

36. Holness CL, Simmons DL. Molecular cloning of CD68, a human macrophage marker related to lysosomal glycoproteins. Blood (1993) 81:1607-13.

37. Rong JX, Shapiro M, Trogan E, Fisher EA. Transdifferentiation of mouse aortic smooth muscle cells to a macrophage-like state after cholesterol loading. Proc Natl Acad Sci U S A (2003) 100:13531-6. doi:10.1073/pnas.1735526100

38. Silva G, Jeney V, Chora A, Larsen R, Balla J, Soares MP. Oxidized hemoglobin is an endogenous proinflammatory agonist that targets vascular endothelial cells. J Biol Chem (2009) 284:29582-95. doi:10.1074/jbc.M109. 045344 
39. Geer JC. Fine structure of human aortic intimal thickening and fatty streaks. Lab Invest (1965) 14:1764-83.

40. Wen Y, Leake DS. Low density lipoprotein undergoes oxidation within lysosomes in cells. Circ Res (2007) 100:1337-43. doi:10.1161/CIRCRESAHA.107. 151704

41. Kurz T, Terman A, Brunk UT. Autophagy, ageing and apoptosis: the role of oxidative stress and lysosomal iron. Arch Biochem Biophys (2007) 462:220-30. doi:10.1016/j.abb.2007.01.013

42. Lee FY, Lee TS, Pan CC, Huang AL, Chau LY. Colocalization of iron and ceroid in human atherosclerotic lesions. Atherosclerosis (1998) 138:281-8. doi:10.1016/S0021-9150(98)00033-1

43. Li W, Ostblom M, Xu LH, Hellsten A, Leanderson P, Liedberg B, et al. Cytocidal effects of atheromatous plaque components: the death zone revisited. FASEB $J$ (2006) 20:2281-90. doi:10.1096/fj.06-6114com

44. Haka AS, Kramer JR, Dasari RR, Fitzmaurice M. Mechanism of ceroid formation in atherosclerotic plaque: in situ studies using a combination of Raman and fluorescence spectroscopy. J Biomed Opt (2011) 16:011011. doi:10.1117/ 1.3524304

45. Balla G, Jacob HS, Balla J, Rosenberg M, Nath K, Apple F, et al. Ferritin: a cytoprotective antioxidant strategem of endothelium. J Biol Chem (1992) 267:18148-53.

46. Balla J, Jacob HS, Balla G, Nath K, Eaton JW, Vercellotti GM. Endothelial-cell heme uptake from heme proteins: induction of sensitization and desensitization to oxidant damage. Proc Natl Acad Sci USA (1993) 90:9285-9.

47. Soe-Lin S, Apte SS, Andriopoulos B Jr, Andrews MC, Schranzhofer M, Kahawita T, et al. Nramp1 promotes efficient macrophage recycling of iron following erythrophagocytosis in vivo. Proc Natl Acad Sci U S A (2009) 106:5960-5. doi:10.1073/pnas.0900808106

48. Pang JH, Jiang MJ, Chen YL, Wang FW, Wang DL, Chu SH, et al. Increased ferritin gene expression in atherosclerotic lesions. JClin Invest (1996) 97:2204-12. doi:10.1172/JCI118661

49. Lane DJ, Merlot AM, Huang ML, Bae DH, Jansson PJ, Sahni S, et al. Cellular iron uptake, trafficking and metabolism: key molecules and mechanisms and their roles in disease. Biochim Biophys Acta (2015) 1853:1130-44. doi:10.1016/j.bbamcr.2015.01.021

50. Musci G, Polticelli F, Bonaccorsi di Patti MC. Ceruloplasmin-ferroportin system of iron traffic in vertebrates. World J Biol Chem (2014) 5:204-15. doi:10.4331/wjbc.v5.i2.204

51. Freeman GJ, Casasnovas JM, Umetsu DT, Dekruyff RH. TIM genes: a family of cell surface phosphatidylserine receptors that regulate innate and adaptive immunity. Immunol Rev (2010) 235:172-89. doi:10.1111/j.0105-2896.2010. 00903.x
52. Gratchev A, Ovsiy I, Manousaridis I, Riabov V, Orekhov A, Kzhyshkowska J. Novel monocyte biomarkers of atherogenic conditions. Curr Pharm Des (2013) 19:5859-64. doi:10.2174/1381612811319330004

53. Habib A, Finn AV. The role of iron metabolism as a mediator of macrophage inflammation and lipid handling in atherosclerosis. Front Pharmacol (2014) 5:195. doi:10.3389/fphar.2014.00195

54. Gomez D, Owens GK. Smooth muscle cell phenotypic switching in atherosclerosis. Cardiovasc Res (2012) 95:156-64. doi:10.1093/cvr/cvs115

55. Rosenfeld ME, Ross R. Macrophage and smooth muscle cell proliferation in atherosclerotic lesions of WHHL and comparably hypercholesterolemic fat-fed rabbits. Arteriosclerosis (1990) 10:680-7. doi:10.1161/01.ATV.10.5.680

56. Nakayama M, Takahashi K, Komaru T, Fukuchi M, Shioiri H, Sato K, et al. Increased expression of heme oxygenase- 1 and bilirubin accumulation in foam cells of rabbit atherosclerotic lesions. Arterioscler Thromb Vasc Biol (2001) 21:1373-7. doi:10.1161/hq0801.093592

57. Lee TS, Lee FY, Pang JH, Chau LY. Erythrophagocytosis and iron deposition in atherosclerotic lesions. Chin J Physiol (1999) 42:17-23.

58. Unruh D, Srinivasan R, Benson T, Haigh S, Coyle D, Batra N, et al. Red blood cell dysfunction induced by high-fat diet: potential implications for obesity-related atherosclerosis. Circulation (2015) 132:1898-908. doi:10.1161/ CIRCULATIONAHA.115.017313

59. Tziakas DN, Chalikias GK, Stakos D, Tentes IK, Papazoglou D, Thomaidi A, et al. Independent and additive predictive value of total cholesterol content of erythrocyte membranes with regard to coronary artery disease clinical presentation. Int J Cardiol (2011) 150:22-7. doi:10.1016/j. ijcard.2010.02.022

60. Pretorius E, Du Plooy JN, Soma P, Keyser I, Buys AV. Smoking and fluidity of erythrocyte membranes: a high resolution scanning electron and atomic force microscopy investigation. Nitric Oxide (2013) 35:42-6. doi:10.1016/j. niox.2013.08.003

Conflict of Interest Statement: The authors declare that the research was conducted in the absence of any commercial or financial relationships that could be construed as a potential conflict of interest.

Copyright $\odot 2017$ Delbosc, Bayles, Laschet, Ollivier, Ho-Tin-Noé, Touat, Deschildre, Morvan, Louedec, Gouya, Guedj, Nicoletti and Michel. This is an open-access article distributed under the terms of the Creative Commons Attribution License (CC BY). The use, distribution or reproduction in other forums is permitted, provided the original author(s) or licensor are credited and that the original publication in this journal is cited, in accordance with accepted academic practice. No use, distribution or reproduction is permitted which does not comply with these terms. 